\title{
Enchentes e saúde pública - uma questão na literatura científica recente das causas, consequências e respostas para prevenção e mitigação
}

\author{
Floods and Public Health - a review of the recent \\ scientific literature on the causes, consequences \\ and responses to prevention and mitigation
}

Carlos Machado de Freitas ${ }^{1}$

Elisa Francioli Ximenes ${ }^{2}$

${ }^{1}$ Centro de Estudos da Saúde do Trabalhador e Ecologia

Humana, Escola Nacional de Saúde Pública, Fundação Oswaldo Cruz. Rua Leopoldo Bulhões 1480/503, Manguinhos. 21.041-210 Rio de Janeiro RJ.

carlosmf@ensp.fiocruz.br

${ }^{2}$ Centro de Estudos e

Pesquisas de Emergências e

Desastres em Saúde,

Fundação Oswaldo Cruz.

\begin{abstract}
Floods are among the most frequent natural disasters and they affect the lives of approximately 102 million people each year, mainly in developing countries and in major urban areas with a tendency to grow further over the coming decades. The scope of this paper is to provide input for a clearer understanding of these events through the results and experiences to be gleaned from the recent scientific literature. From the Pubmed database, 70 articles were analyzed that fulfilled the criteria to address at least one of the items selected for analysis, namely: 1) causes; 2) consequences; 3) responses and actions: submission of proposals and solutions for the prevention and/or mitigation of the risks and impacts of flooding. Tables for each of the items selected were organized in order to systematize and synthesize the results for causes (attributed to natural and human activities); environmental, infrastructure and services, and health consequences (injuries and diseases classified according to chapters of ICD-10); prevention and mitigation responses and actions. It was concluded that given the scenarios of increased frequency and severity of these events, the challenges facing public health for disaster risk reduction require integrated responses with broad policies for sustainable development. Key words Floods, Public health, Environmental health, Natural disasters
\end{abstract}

Resumo As enchentes são os desastres naturais com maior frequência e afetam a vida de aproximadamente 102 milhões de pessoas a cada ano, principalmente nos paises em desenvolvimento e em grandes centros urbanos, com tendência de aumento nas próximas décadas. O objetivo é oferecer subsídios para uma melhor compreensão destes eventos, através dos resultados e experiências encontrados na literatura científica recente. Por meio de busca no Pubmed foram analisados 70 trabalhos aos quais se teve acesso e se enquadraram nos critérios de abordar pelo menos um dos itens selecionados para análise, que eram: causas; consequências; respostas e ações; encaminhamento de propostas e soluções para a prevenção el ou mitigação dos riscos; $e$, impactos das enchentes. A partir destes critérios foram montados quadros para cada um dos itens de análise de modo a sistematizar e sintetizar os resultados para as causas, as consequências ambientais, a infraestrutu$r a$, os serviços e a saúde e para as respostas e ações de prevenção e mitigação. Considerou-se que, dados os cenários de aumento na frequência e gravidade destes eventos, os desafios para o setor saúde para a redução de riscos de desastres exigem respostas integradas com amplas politicas para o desenvolvimento sustentável.

Palavras-chave Enchentes, Inundações, Saúde pública, Saúde ambiental, Desastres naturais 
Introdução

Dentre os inúmeros impactos ambientais que afetam a vida de milhões de pessoas no mundo, os eventos como enchentes atingem cerca de 102 milhões de pessoas por ano ${ }^{1}$ e a maior parte das populações expostas (95\%) e dos óbitos (95\%) se encontram nos países de menor renda per capita (igual ou menor que 3.705 dólares por ano) ${ }^{2}$. Seus impactos são mais severos para determinados grupos populacionais e espaços geográficos mais vulneráveis, seja nos países mais pobres ou mesmo nos países mais ricos, como foi demonstrado após o furacão Katrina.

Suas causas e consequências, bem como as respostas e as ações para prevenção e mitigação vêm se tornando temas de grande interesse após inúmeros alertas da comunidade científica sobre o potencial de mudanças ambientais em larga escala, como as alterações climáticas, resultarem no aumento da frequência e intensidade das chuvas e de outros eventos extremos ${ }^{3-9}$.

Neste artigo foi realizada uma revisão da literatura sobre a relação enchentes e saúde pública, de modo a oferecer subsídios para uma melhor compreensão destes eventos, que correspondem a cerca de $60 \%$ dos desastres naturais registrados no país e 70\% na América Latina e Caribe. Através dos resultados e experiências encontrados na literatura científica recente foram abordados três aspectos que devem ser compreendidos de modo integrado: as causas, as consequências e as respostas e ações para prevenção e mitigação dos riscos e impactos. Esta revisão pode ser considerada oportuna, uma vez que os desastres naturais ocupam cada vez mais a agenda científica e governamental do país, sendo exemplos a criação do Centro Nacional de Monitoramento e Alerta aos Desastres Naturais pelo Ministério de Ciência e Tecnologia e da Força Nacional do Sistema Único de Saúde pelo Ministério da Saúde.

\section{Metodologia}

Foi realizada revisão de literatura, de artigos disponíveis no PubMed até o ano de 2011, por ter sido considerada a base bibliográfica mais abrangente e ao mesmo tempo específica para a saúde pública. Por se tratar de uma base bibliográfica na língua inglesa, foram escolhidos como descritores gerais os termos: "floods" and "public health". O objetivo foi a capturar do maior número de artigos que abordassem o tema das enchentes, e ao mesmo restringir àqueles que estabelecessem um vínculo mais direto entre estes eventos e a saúde pública, compreendida aqui como uma resposta do estado as necessidades de saúde.

Foram identificados 114 artigos, os quais foram submetidos a dois grupos de critérios de inclusão. O primeiro grupo foi estar em um idioma que pudesse ser lido pelos autores, sendo estes: inglês, português ou espanhol. A partir deste critério 9 artigos não foram incluídos (5 em chinês, 2 em francês, 1 em Norueguês e 1 em Russo). O segundo grupo era o artigo abordar no resumo pelo menos um dos itens selecionados para análise, que eram: (1) causas; (2) consequências; (3) respostas e ações: encaminhamento de propostas e soluções para a prevenção e/ou mitigação dos riscos e impactos das enchentes. Foram selecionados 74 artigos e excluídos outros 31, que abordavam temas bastante específicos de investigações de doenças relacionadas às enchentes. Destes 74 só se teve acesso direto à 62 em revistas que disponibilizam o acesso público aos artigos ao através das revistas disponíveis no sítio de periódicos da Capes. Outros 12 foram solicitados, tendo chegado apenas 8 via Comut, restando ao final 70 artigos para análise, sendo o primeiro publicado em 1949 e o último em 2011.

\section{Resultados}

\section{Causa das enchentes}

Dos 70 artigos, 27 indicavam causas para as enchentes sistematizadas no Quadro 1. Destes, 12 apontaram as mudanças climáticas e o aquecimento global como causas ${ }^{5-16}$ e 5 o aumento de chuvas intensas e localizadas ${ }^{14,17-20}$. Fenômenos relacionados às mudanças climáticas e que influenciam as enchentes são: furacões, ciclones tropicais e monsões ${ }^{19,21}$, derretimento intensivo de neve e geleiras ${ }^{6,19,20,22-26}$ e a Oscilação Sul - El Niño ${ }^{27,28}$. Também foram apontadas outras causas, como tsunamis ${ }^{16,21,24,29}$, bem como questões relacionadas ao uso e ocupação do solo; descarte inadequado de lixo; intensificação da agricultura; construções de barragens e hidrelétricas; desmatamento ${ }^{13}$; erosão do solo resultando no assoreamento dos $\operatorname{rios}^{6,30}$ assim como a rápida urbanização sem planejamento adequado ${ }^{16}$.

\section{Consequências das enchentes}

Dos 70 artigos, 51 indicaram consequências das enchentes, sistematizadas nos Quadros 2, 3 e 4, classificadas do modo a seguir: (1) consequên- 
Quadro 2. Consequências ambientais das enchentes

\begin{tabular}{|l|l|}
\hline $\begin{array}{l}\text {. Contaminação biológica da água para consumo humano e } \\
\text { alimentos } \\
\text {. Contaminação química da água para consumo humano e solos }\end{array}$ & $\begin{array}{l}\text { Contaminação de água, solo e } \\
\text { alimentos }\end{array}$ \\
\hline $\begin{array}{l}\text {. Comprometimento da rede e fontes alternativas de } \\
\text { abastecimento de água, dos serviços de coleta e tratamento de } \\
\text { esgoto, bem como dos serviços de coleta e disposição do lixo }\end{array}$ & $\begin{array}{l}\text { Comprometimento dos serviços de } \\
\text { saneamento ambiental }\end{array}$ \\
\hline $\begin{array}{l}\text {. Alteração nos ciclos dos vetores, hospedeiros e reservatórios de } \\
\text { doenças e nas formas de exposições ambientais dos humanos }\end{array}$ & $\begin{array}{l}\text { Alteração nos ciclos ecológicos e } \\
\text { exposições humanas }\end{array}$ \\
\hline
\end{tabular}

cias ambientais; (2) consequências sobre a saúde - mortalidade e morbidade; (3) consequências sobre a infraestrutura, serviços e economia local.

\section{Consequências ambientais}

Mais da metade, 27 artigos, destacaram as consequências ambientais, as quais foram sistematizadas no Quadro 2.

A rede de distribuição de água para consumo humano ${ }^{31-37}$ quando afetada, compromete o fornecimento para as casas e mesmo para os serviços de saúde ${ }^{25,31,33}$, resultando, entre outras coisas, na falta de água potável para a população ${ }^{34}$. Além de afetar a distribuição, a água da rede utilizada pela população, bem como poços e nascentes, acabam sendo contaminados por agentes infecciosos presentes nas águas das enchentes ${ }^{4,8,12,16,21,23,24,33-38}$, aumentando o risco de doenças transmitidas pela ingestão da água ${ }^{35,36,39}$.

A contaminação de alimentos armazenados em casas ou mercados, bem como de organismos marinhos filtradores, como ostras e mariscos, é também relatada como um dos problemas ambientais ${ }^{21,32,34,36,38,40}$. Outra forma de contami-
Causas atribuídas aos fenômenos e eventos "naturais"

Causas atribuídas as atividades humanas 
Quadro 3. Consequências sobre a saúde provocadas pelas enchentes

\begin{tabular}{|c|c|}
\hline Agravos e doenças, incluindo alguns sinais e sintomas & $\begin{array}{l}\text { Capítulos da Classificação } \\
\text { Internacional de Doenças } 10\end{array}$ \\
\hline $\begin{array}{l}\text { Diarreias e gastroenterites } \\
\text {. Cólera } \\
\text {. Febre tifóide } \\
\text {. Varíola } \\
\text {. Hepatites A } \\
\text {. Hepatites E } \\
\text {. Poliomielite } \\
\text {. Malária } \\
\text {. Febre amarela } \\
\text {. Dengue } \\
\text {. Encefalite de St Louis } \\
\text {. Filariose linfática } \\
\text {. Leptospirose } \\
\text {. Esquistossomose } \\
\text {. Shiguelose } \\
\text {. Escherichia coli [E. Coli] } \\
\text {. Giardíase }\end{array}$ & $\begin{array}{l}\text { Capítulo I - Doenças infecciosas e } \\
\text { parasitárias }\end{array}$ \\
\hline . Desnutrição & $\begin{array}{l}\text { Capítulo IV - Doenças endócrinas, } \\
\text { nutricionais e metabólicas }\end{array}$ \\
\hline $\begin{array}{l}\text { Estados de estresse pós-traumático } \\
\text { Transtornos de adaptação } \\
\text {. Transtornos não orgânicos do sono } \\
\text {. Insônia } \\
\text {. Pesadelos e memórias repetidas sobre o evento } \\
\text {. Amnésia } \\
\text {. Dificuldade de concentração } \\
\text {. Irritabilidade e raiva } \\
\text { Fobias, ansiedade e pânico, depressão, perda do apetite, fadiga, } \\
\text { dificuldade de concentração, tontura } \\
\text {. Abuso no consumo de álcool e medicamentos } \\
\text {. Transtornos do comportamento e emocionais durante a infância } \\
\text { Úlceras }\end{array}$ & $\begin{array}{l}\text { Capítulo V - Transtornos mentais } \\
\text { e do comportamento }\end{array}$ \\
\hline . Conjuntivites & $\begin{array}{l}\text { Capítulo VII - Doenças do olho e } \\
\text { anexo }\end{array}$ \\
\hline . Pressão arterial alta & $\begin{array}{l}\text { Capítulo IX - Doenças do } \\
\text { aparelho circulatório }\end{array}$ \\
\hline $\begin{array}{l}\text { Rinite alérgica } \\
\text {. Infecções respiratórias agudas } \\
\text { Sinusites severas } \\
\text {. Asmas } \\
\text { - Infecções pulmonares } \\
\text {. Síndrome tóxica da poeira orgânica } \\
\text {. Laringite }\end{array}$ & $\begin{array}{l}\text { Capítulo X - Doenças do aparelho } \\
\text { respiratório }\end{array}$ \\
\hline . Dermatites e erupções cutâneas & $\begin{array}{l}\text { Capítulo XII - Doenças da pele e } \\
\text { do tecido subcutâneo }\end{array}$ \\
\hline . Distensões musculares & $\begin{array}{l}\text { Capítulo XIII - Doenças do } \\
\text { sistema osteomuscular e do tecido } \\
\text { conjuntivo }\end{array}$ \\
\hline
\end{tabular}

continua 


\begin{tabular}{l}
\hline Quadro 3. continuação \\
\begin{tabular}{|l|l|}
\hline \multicolumn{1}{|c|}{ Agravos e doenças, incluindo alguns sinais e sintomas } & \multicolumn{1}{c|}{$\begin{array}{c}\text { Capítulos da Classificação } \\
\text { Internacional de Doenças } \mathbf{1 0}\end{array}$} \\
\hline . Infecções renais & $\begin{array}{l}\text { Capítulo XIV - Doenças do } \\
\text { aparelho geniturinário }\end{array}$ \\
\hline $\begin{array}{l}\text { - Asfixia } \\
\text { Intoxicações e envenenamentos } \\
\text { Hipotermia } \\
\text { Lesões, traumatismos, cortes, lacerações e ferimentos }\end{array}$ & $\begin{array}{l}\text { Capítulo XIX - Lesões, } \\
\text { envenenamentos e algumas outras } \\
\text { conseqüências de causas externas }\end{array}$ \\
\hline $\begin{array}{l}\text { Violência doméstica } \\
\text { Choques elétricos } \\
\text { Afogamentos } \\
\text { Quedas }\end{array}$ & $\begin{array}{l}\text { Capítulo XX - Causas externas de } \\
\text { morbidade e de mortalidade }\end{array}$ \\
\hline
\end{tabular}
\end{tabular}

Quadro 4. Consequências para a infraestrutura local, serviços, economia e sociedade local

\begin{tabular}{|l|l|}
\hline $\begin{array}{l}\text {. Interrupção total ou parcial de pontes, ruas e estradas por } \\
\text { inundação ou destruição } \\
\text {. Rompimento de diques de contenção } \\
\text {. Rompimento de tanques de combustíveis } \\
\text {. Curto-circuito elétrico }\end{array}$ & $\begin{array}{l}\text { Consequências sobre infraestrutura } \\
\text { local }\end{array}$ \\
\hline $\begin{array}{l}\text {. Interrupção total ou parcial do fornecimento de serviços de } \\
\text { eletricidade, gás e comunicação } \\
\text {. Interrupção total ou parcial do funcionamento de escolas, } \\
\text { comércio, serviços funerários e de saúde }\end{array}$ & Consequências sobre os serviços locais \\
\hline $\begin{array}{l}\text {. Comprometimento total ou parcial das atividades agrícolas e } \\
\text { pecuárias } \\
\text {. Prejuízos econômicos pela destruição total ou parcial de } \\
\text { propriedades, casas e construções } \\
\text { Prejuízos econômicos pela destruição total ou parcial das } \\
\text { fontes de renda e trabalho } \\
\text {. Perdas de bens pessoais e de valor sentimental }\end{array}$ & $\begin{array}{l}\text { Prejuízos econômicos e perdas } \\
\text { materiais }\end{array}$ \\
\hline $\begin{array}{l}\text { Rompimento ou fortalecimento da amizade, cooperação e } \\
\text { laços afetivos entre os membros de uma comunidade afetada }\end{array}$ & $\begin{array}{l}\text { Rompimento ou fortalecimento das } \\
\text { relações sociais locais }\end{array}$ \\
\hline
\end{tabular}

águas residuais, lixo, silte e material em decomposição que recobrem as ruas e os solos após as enchentes ${ }^{4,21,33,36}$. Com o ambiente alterado, há um considerável aumento na quantidade de mosquitos, moscas e ratos, assim como aumento da contaminação fecal por parasitos e helmin$\operatorname{tos}^{4,21,31,33,34,43,44}$. E, mesmo em situações onde não se diagnosticou aumento na população de vetores, hospedeiros e reservatórios, identificou-se o potencial aumento na exposição devido ao desalojamento da população ${ }^{4,36}$.

\section{Consequências sobre a saúde - mortalidade e morbidade}

Dos 51, 44 apontaram mortalidade e morbidade entre as principais consequências das enchentes, organizadas a partir da Classificação Internacional de Doenças no Quadro 3.

Em relação à mortalidade, 20 apontaram os óbitos durante as enchentes como principal consequência ${ }^{4,6-8,11-14,16,24,31,33-36,45-49}$, sendo especificados os por diarréias ${ }^{7,12,13,46,50}$ e os por hipoter- 
mia ${ }^{16,35,51}$, bem como por lesões ${ }^{8,16}$ e afogamen$\operatorname{tos}^{8,11,16,35,51}$. Alguns estudos apontaram para um aumento nas taxas de mortalidade nos 12 meses após a ocorrência da enchente ${ }^{7,11,18}$, demonstrando que os óbitos não se limitam aos imediatamente após os eventos. Além das mortes de humanos, há também indicações das de animais ${ }^{38}$, de modo que suas carcaças nas ruas e casas, após o pico das enchentes ${ }^{33}$, também se convertem em fonte de doenças.

Em relação à morbidade, 11 apontaram que existe o aumento na incidência de doenças potencialmente epidêmicas nos períodos após as enchentes $^{52}$, como o aumento de transmissíveis ${ }^{4,11,16,18}$, que podem ser transmitidas através da água e alimentos contaminados ${ }^{4,7,21,38,42}$, assim como por vetores e hospedeiros de doenças $^{4,21,32}$, principalmente nos países mais pobres ${ }^{5,8}$.

Dentre as doenças transmitidas através da água e alimentos contaminados foram especificadas as seguintes: gastroenterites ${ }^{4,8,11,14,17,18,21,46,47}$, ${ }^{50}$, e infecções por E. Coli, Giárdia e Shigella ${ }^{12}$, cólera $^{12,21,50}$, febre tifóide ${ }^{12,21}$, varíola ${ }^{52}$, hepatites $\mathrm{A}^{12,35}$ e E, e poliomelite ${ }^{21,50}$. Dentre as doenças transmitidas por vetores e hospedeiros foram especificadas: malária ${ }^{7,13,21,35,47,50}$; febre amarela ${ }^{35}$; febre hemorrágica ${ }^{50}$, dengue en, $^{14,47,50}$; encefalite de St Louis $^{21}$; filariose linfática ${ }^{21}$; e leptospiro$\mathrm{se}^{21,36,42,47,53}$.

Além destas doenças, em grande parte relacionadas ao saneamento ambiental inadequado, há também o aumento da umidade neste mesmo período, com rápido crescimento de micobacterias (como Mycobacterium marinum) ${ }^{21}$ e proliferação de fungos ${ }^{54,55}$, agravando os problemas de saúde para as pessoas alérgicas e susceptíveis ${ }^{4}$ tendo sido registrados rinite alérgica ${ }^{42}$, infecções respiratórias agudas $4,5,8,11,14,16,21,35,38,46,50$, asmas $^{14,18,42,}$, sinusites severas ${ }^{18,42}$, infecções pulmonares ${ }^{18}$, síndrome tóxica da poeira orgânica ${ }^{42}$, dermatites ${ }^{14,17,18,21,45,50}$ e conjuntivites ${ }^{56}$.

Outras doenças encontradas na literatura no período após as enchentes são: laringite, pressão alta e infecções renais ${ }^{18}$. Foram também citados sintomas como: tosses, resfriados e gripes, dores de ouvido e garganta ${ }^{18}$, dores abdominais, dores no peito e palpitações ${ }^{46}$, dores de cabeça ${ }^{18,46}$, náuseas e enjôos ${ }^{18,46}$ e febres ${ }^{17,43,46,47}$.

Além das doenças envolvendo agentes biológicos, a literatura também aponta para os impactos sobre a saúde mental ${ }^{16}$ e emocional das populações expostas às enchentes. Estados de estresse pós-traumático ${ }^{14,44,50,51,57-59}$ e transtornos de adaptação ${ }^{18}$ foram indicadas como algumas das consequências apresentando percentuais entre $10 \%$ a $25 \%$ entre os expostos, afetando mais intensamente determinados grupos sociais como as mulheres, os moradores de áreas rurais, os analfabetos, as crianças, os idosos, os deficientes e os mais pobres ${ }^{11,18,45}$.

Foram especificadas transtornos e síndromes devido a fatores emocionais, como distúrbios no sono, insônia, pesadelos e memórias repetidas sobre o evento, amnésia, dificuldade de concentração, irritabilidade e raiva ${ }^{45,59}$, ansiedade ${ }^{58,59}$, fobias, pânico, depressão $0^{50,58,59}$, perda de apetite, fadiga, tonteiras ${ }^{7-9,11,39,45,46}$ e suicídio ${ }^{59}$. Grande parte destas consequências se manifesta após as enchentes e principalmente durante períodos de chuvas $^{18}$, mas há relatos que apontam também para as mesmas relacionadas à quebra da rotina familiar e social e durante o período de reconstrução (limpezas, reparos, acionamento de seguros, indenizações ou assistência social) $)^{4,11,18,24,39}$. Outra fonte de ansiedade e preocupação relatada entre os afetados é a suspeita de possível contaminação na família por produtos químicos ${ }^{18}$, especialmente em locais industriais ${ }^{39}$, bem como o medo de ratos e as doenças causadas pelo mesmo (leptospirose) e outras como hepatite e febre tifóide ${ }^{18}$. A falta de orientações e informações ou a presença de orientações conflitantes e incompatíveis vinda das autoridades em saúde ${ }^{18}$, assim como a falta de vacinação também contribui para elevar a ansiedade entre a população exposta ${ }^{18}$. Além destes, foram apontadas outras consequências sobre a saúde mental e emocional nas populações expostas, como violência familiar e abuso no consumo de álcool e medicamentos entre adultos ${ }^{18,24}$ e distúrbios de comportamento em crianças ${ }^{7}$ e jovens meninas expostas a assédio mental e sexual em abrigos temporários $^{59}$. Estas consequências mentais e emocionais podem durar meses ou anos após as enchentes, reaparecendo sempre que fortes chuvas ou outras enchentes ocorrerem novamente ${ }^{18}$.

Há também um conjunto de outras consequências que podem ocorrer imediatamente após as enchentes, constituindo também uma grande fonte de exposição ao risco de acidentes, lesões ${ }^{12-}$ ${ }^{14,49,51}$ e doenças, principalmente para os que se tornam desalojados ou desabrigados, apontado em 10 artigos $^{4,8,11,21,24,33,34,52,60,61}$. Ambas condições implicam no deslocamento massivo de populações que podem ficar aglomeradas em abri$\operatorname{gos}^{31,34,47,61-63}$ (onde muitas vezes a aglomeração com precária infraestrutura cria condições para proliferação de doenças transmissíveis) ou mesmo tornarem-se refugiados ambientais em situações mais extremas ${ }^{8}$. Durante o deslocamento em 
massa são relatados ferimentos e lesões, incluindo lacerações, úlceras e erupções cutâneas causadas por infecções ${ }^{4,11,14,17,24,32,35,45,46}$. Comunidades inteiras podem ficar isoladas e deixam de ter acesso ao transporte, serviços de saúde, serviços emergenciais, alimento, água potável e necessidades básicas de sobrevivência ${ }^{34}$.

Mesmo para os que retornam para suas casas, o reparo e a limpeza de casas e ruas por pessoas não preparadas para alguns destes tipos de trabalho pode resultar em choques elétricos ${ }^{16}$, asfixia (em pessoas consertando e limpando seus poços artesianos), distensões musculares, cortes e intoxicações por monóxido de carbono (em pessoas que utilizam gasolina em geradores de eletricidade) e envenenamentos por picadas de cobra $^{11,16,24,32,35,51}$.

Além destas consequências foram também relatados potenciais efeitos genotóxicos, citotóxicos, mutagênicos e carcinogênicos relacionados a contaminação das águas por agrotóxicos e metais pesados ${ }^{8,39,41}$.

\section{Consequências sobre a infraestrutura, serviços e economia local}

Este tema esteve presente em 29 dos 51 artigos, demonstrando sua importância para a compreensão das consequências ambientais e sobre a saúde e sistematizados no Quadro 4.

Em situações de enchente, pontes, ruas e estradas podem ser destruídas ou inundadas impedindo o acesso dos e aos serviços de emergência, entre estes os de saúde $e^{6,21,31,34}$. A força da água das enchentes pode destruir diques de contenção ${ }^{52}$, como no caso do Furacão Katrina em Nova Orleans (EUA), incêndios a partir do rompimento de tanques de combustíveis ${ }^{26}$ e curtocircuito elétrico ${ }^{33}$ podendo ocorrer explosões e queimaduras ${ }^{16}$. O comprometimento parcial ou total do fornecimento de energia elétrica local, por conseguinte, afeta os serviços de telefones públicos e privados, de fornecimento de gás e água $^{8,18,21,31,34,38}$, comprometendo desde o armazenamento de alimentos e vacinas, até a higiene pessoal dos moradores locais ${ }^{24}$. Serviços de distribuição de água e de saneamento podem ser seriamente comprometidos ${ }^{26}$. A depender da magnitude do evento, escolas e comércio poderão ter suas atividades interrompidas por me$\mathrm{ses}^{38}$. Também há relato de impactos de enchentes em cemitérios, fazendo flutuar na água da inundação mais de 400 caixões $^{31}$.

Durante as enchentes os serviços de saúde também podem ser afetados ${ }^{8,13,16,24,32,33,51}$, tanto por ter as instalações atingidas e equipamentos e materiais de primeiros socorros comprometidos a partir do contato com a água das enchentes ${ }^{48}$, como pelo aumento da procura dos serviços pela população imediatamente após as mesmas ${ }^{18}$. Não só o cotidiano dos serviços de saúde é alterado (marcação de consultas, por exemplo), como também podem ser comprometidos programas de combate a doenças transmitidas por vetores, vacinação e de tratamento de doenças ${ }^{64}$, como a tuberculose $e^{4,17,18,21}$.

As enchentes podem também gerar consequências sobre a agricultura ${ }^{13}$ e a pecuária ${ }^{25,52}$, atingindo a produção de alimentos e a qualidade do mesmo, como a do leite, por exemplo ${ }^{61}$, agindo diretamente a condição nutricional da população afetada ${ }^{60,61}$ podendo levar à desnutrição e à fome $\mathrm{e}^{13,14,16,51 \text {, }}$

Além de atingir a infraestrutura e os serviços há também prejuízos econômicos e perdas materiais ${ }^{33,34,37,38,45,47,60}$ que impactam a economia local ${ }^{5,8,9,14}$. Os prejuízos envolvem propriedades, casas e construções que são parcial ou totalmente destruídas ${ }^{6,13,24,33,48}$, resultando em desalojados e desabrigados, afetando suas fontes de renda e trabalho no comércio, fábricas, plantações e criação de animais ${ }^{21,25,33,34,60}$. Há ainda perdas materiais incomensuráveis como os bens pessoais e de valor sentimental ${ }^{11,18,38}$.

Um artigo apontou para o fortalecimento dos laços afetivos e de cooperação entre os membros da comunidade afetada ${ }^{18}$.

\section{Respostas e ações para prevenção e mitigação}

Dos 70 artigos revisados, 41 abordaram as respostas sociais às enchentes, sistematizadas no Quadro 5 e classificadas do modo a seguir: (1) Monitoramento socioambiental de vulnerabilidade e vigilância em saúde como instrumentos para prevenção e melhoria das respostas; (2) capacidade de avaliações rápidas; (3) capacidade de respostas do setor saúde.

\section{Monitoramento socioambiental de vulnerabilidade e vigilância em saúde como instrumentos para prevenção e melhoria das respostas}

Foram encontrados 13 que abordaram o monitoramento do clima e da vulnerabilidade social e ambiental, assim como sistemas de vigilância em saúde (Quadro 5).

Para a prevenção de curto prazo das consequências das enchentes foram apontados o mo- 
Quadro 5. Respostas e ações para prevenção e mitigação

. Monitoramento do clima e previsão meteorológica acopladas a um sistema de alerta eficaz

. Monitoramento da vulnerabilidade em relação às condições de saneamento, saúde e de ocupação em áreas de risco

. Estruturação de sistemas de monitoramento e vigilância sobre as enchentes (eventos), áreas e grupos vulneráveis, e suas consequências para a saúde, permitindo aprendizado contínuo para a preparação e as respostas às necessidades de saúde pósenchentes (curto, médio e longo prazos)

- Avaliação das necessidades de evacuações das áreas acoplada à organização de abrigos e alojamentos temporários

imediatamente no período pós-enchente e de casas temporárias para os que perderam suas habitações

- Avaliação do sistema de abastecimento de água e da qualidade da mesma acoplado ao rápido tratamento e fornecimento de água potável para população

- Avaliação das áreas em que o sistema de coleta e de disposição do lixo, bem como de coleta e de tratamento de esgoto foram mais afetados acoplados a ações rápidas de coleta e disposição do lixo, reparo do sistema de coleta e tratamento de esgoto e enterro de animais mortos

- Avaliação dos alimentos disponíveis e descarte dos contaminados acoplada à distribuição de mantimentos, utensílios de cozinha e alimentos para os desalojados, bem como higienização dos mesmos

- Avaliação do impacto sobre serviços fundamentais para as crianças, como creches e escolas, acoplada ao restabelecimento rápido dos mesmos

. Controle de vetores de hospedeiros de doenças, do nível individual ao coletivo

. Intensificação das ações de vigilância em saúde da exposição da população aos riscos de doenças e dos efeitos sobre a saúde

. Vacinação rápida para o risco de doenças para a população nas áreas afetadas

. Provisão de serviços (tendas de saúde, hospitais de campanha, laboratórios) e de pessoal de saúde (médicos, enfermeiras, terapeutas, técnicos de laboratório) extras nas áreas afetadas . Ações de cuidados e apoio psicossocial às populações afetadas para prevenir e monitorar os efeitos de longo prazo dos Transtornos do Estresse Pós Traumático

. Programas de educação em desastres e saúde para a população (riscos das enchentes, alertas, evacuações, potenciais impactos sobre a saúde, primeiros socorros e ressuscitação, ações necessárias para prevenir doenças)

. Programas de treinamento para profissionais de saúde de áreas vulneráveis ou afetadas, com ênfase em primeiros socorros, tratamento e prevenção de doenças relacionadas às enchentes - Planejamento de ações conjuntas e coordenadas do setor saúde com outros setores governamentais (defesa civil, meio ambiente, forças armadas, agricultura, comunicação, transportes, polícia, etc.) e em diferentes níveis (municipais, estaduais e federais), além de empresas privadas (comunicação, transportes, etc.) e organizações não governamentais

. Medidas estruturais de reparos e reconstrução de instalações e vias de comunicação, assim como de habitações

Monitoramento socioambiental e vigilância em saúde como instrumentos para prevenção e melhoria das respostas

Capacidade de avaliações rápidas das áreas afetadas para identificar impactos, riscos e necessidades sociais, ambientais e de saúde da população afetada

Capacidade de respostas do setor saúde (vigilância em saúde, controle de hospedeiros e vetores de doenças, vacinações, atenção e cuidado, educação e conscientização em relação aos riscos e problemas de saúde) 
nitoramento do clima e a previsão meteorológi$\mathrm{ca}^{65}$, unidas a um sistema de alerta eficaz ${ }^{4,24}$. Para a prevenção de médio e longo prazos das enchentes foi apontado o estudo ${ }^{37}$ e o monitoramento da vulnerabilidade, particularmente das condições sociais (acesso a saneamento ambiental e a serviços de saúde de qualidade) e de ocupação da população em áreas de risco, de modo a identificar as intervenções necessárias, como planejamento sobre o uso e a ocupação do solo, para a diminuição das consequências destes eventos nas populações ${ }^{2,5,114,7,18}$.

Para alguns autores, a formulação de políticas públicas para prevenção e respostas as enchentes $^{12,14}$ exige a estruturação de sistemas de monitoramento e vigilância sobre estas (eventos), as áreas e os grupos vulneráveis, e suas consequências para a saúde, permitindo melhor preparação (incluindo desenvolvimento e revisão dos planos de emergências) para respostas mais rápidas e efetivas as necessidades de saúde pósenchentes ${ }^{3,4,8,11,24,32,39}$.

\section{Capacidade de avaliações rápidas e de atenção às necessidades}

Neste tema foram encontrados 16 artigos abordando o mesmo como fundamental para respostas rápidas (Quadro 5).

A avaliação imediata das áreas afetadas deve ser realizado para identificar principais impactos, danos e prejuízos à infraestrutura (pontes, ruas, estradas, habitações) e serviços (saúde, água, esgotamento sanitário, lixo), assim como os principais riscos à saúde, a morbidade e a mortalidade, bem como necessidades de saúde da população afetada $7,32-34,39,48,60$.

Alguns consideram que esta avaliação deve propiciar de imediato a organização de abrigos e alojamentos temporários logo no período pós-enchente para os desalojados e desabrigados ${ }^{20,33,38,48}$.

Em relação aos serviços, um dos cuidados fundamentais após enchentes é com a qualidade da água para consumo humano ${ }^{20}$, já que esta pode ser fonte de muitas doenças, incluindo: 1) monitoramento e reparo nos encanamentos de distribuição de água; 2) monitoramento e análises da qualidade distribuída e consumida nos locais afetados ${ }^{31-33,38}$, incluindo a contaminação por produtos químicos ${ }^{39}$; 3) tratamento ${ }^{11,30,47,52}$, incluindo a distribuição de cloro para a população afetada tratar a mesma ${ }^{13,3,34,38,52}$; 4) fornecimento e distribuição de água potável à população $0^{33,38,52}$, principalmente em zonas em que a água consumida é de $\operatorname{poços}^{32}$; 5) campanhas para racionalização do consumo de água ${ }^{33}$.
Operações de limpeza dos destroços e resíduos sólidos (pedaços de móveis, veículos, destroços de construções, etc. $)^{11,20,34,52}$, principalmente nas habitações e prédios públicos ${ }^{38}$, com destinação final adequada e rápida para prevenir, entre outras coisas, casos de leptospirose e picadas por cobras ${ }^{32}$. O enterro de animais mortos também deve ser imediatamente providenciado $^{38}$. Outros artigos apontam para a necessidade de reparo imediato do sistema de coleta e tratamento de esgoto ${ }^{11,20,25,52}$.

Em relação à segurança alimentar duas medidas aparecem como fundamentais e conectadas. A primeira é a distribuição de alimentos e utensílios de cozinha para os desabriga$\operatorname{dos}^{33,34,38,48}$, de preferência com acompanhamento de um nutricionista ${ }^{38}$. A segunda é a promoção da higienização dos alimentos existentes ${ }^{40}$ e o descarte de alimentos contaminados ${ }^{33}$.

Por fim, particularmente em relação às crianças, além dos cuidados em saúde, o apoio envolve disponibilidade de locais como creches e escolas onde possam ficar enquanto os pais estão ocupados no processo de recuperação pós-enchente (mutirões de limpeza e reparos) $)^{18,38}$.

Capacidade de respostas do setor saúde

A capacidade de respostas do setor saúde envolveu o maior número de artigos sobre as respostas sociais, totalizando 32 (Quadro 5).

Dentre as respostas, o controle de vetores, através da distribuição de inseticidas para combate aos mosquitos ${ }^{4,11,34,38,52,60}$, solicitação de ajuda externa (outros estados ou nível federal) (1,33 $^{31}$ ou mesmo aplicações por aviões em grandes áreas $^{52}$ surge como um primeiro tema.

Um segundo é a necessidade de intensificação das ações de vigilância em saúde da exposição da população aos riscos de doenças (água, alimentos, lixo, esgotos, contaminantes químicos, vetores e hospedeiros de doenças, etc.), dos efeitos sobre a saúde (mortalidade e morbidade) e investigação epidemiológica de possíveis surtos de doenças (febre tifóide, diarréias, malária, etc.) nos locais atingidos pelas enchentes ${ }^{4,11,24,32,35,39,48,52,61}$ de modo a subsidiar as ações emergenciais de controle e prevenção, bem como de tratamento e recuperação em saúde 4,17,20,34,52. $^{\text {. }}$

A vacinação surge como outro tema, amplamente adotada nos períodos após as enchentes, sendo as mais utilizadas as vacinas contra para tétano, febre tifóide, ${ }^{66}$ e cólera ${ }^{33-35,38,50,52}$ e, antes da erradicação, a varíola ${ }^{36,52}$.

Um quarto tema e que aparece para os casos mais graves é o de provisão de serviços e pessoal 
de saúde extras para a população afetada ${ }^{66}$. Em termos de infraestrutura física, são apontados tendas de saúde, hospitais, bancos de sangue e laboratórios de emergência para atender o maior número de pessoas possíveis, seja pelo aumento da demanda, seja pela destruição parcial ou total da infraestrutura de saúde $\mathrm{e}^{17,34,38}$, além de serviços de saúde nas cidades ou municípios vizinhos oferecerem leitos extras ${ }^{7}$. Em relação ao pessoal, são apontados principalmente médicos, enfermeiros, terapeutas, e técnicos de laboratório, assim como estudantes de graduação na área da saúde, tanto de serviços públicos, como de outras organizações da sociedade civil ${ }^{17,20,31,33,34}$.

Outro tema importante se refere às ações de cuidado e apoio psicossocial às populações afetadas para prevenir (envolvendo ações de comunicação nas etapas de planejamento de emergências e pós-enchentes) e monitorar os efeitos de longo prazo dos Transtornos do Estresse Pós Traumático ${ }^{4,732,45,47}$, com visita de profissionais da área de saúde e social aos grupos populacionais que podem ser considerados mais vulneráveis (crianças, idosos, grávidas, deficientes) $)^{11,18,20,34}$.

Além destes temas diretamente relacionados ao controle de vetores, prevenção de doenças através de vacinas, intensificação das ações de vigilância em saúde, provisão de recursos extras (humanos e materiais) em saúde e apoio psicossocial, surgiram outros que exigem ações do setor saúde com outros setores.

O primeiro é a questão da educação (em saúde) para os desastres. Há o consenso que este tema deve ser desenvolvido principalmente para as comunidades que se encontram sob risco de enchentes e os grupos vulneráveis, tanto sobre como lidar com estes eventos (programas de comunicação, alertas e evacuações) e seus riscos, como sobre a recuperação após os mesmos, fortalecendo a capacidade de resiliência ${ }^{4,7,19,46,52,67,68}$. Os programas de educação para desastres podem ser de médio ou longo prazos, permitindo construir mapas mentais que auxiliem no planejamento preventivo e de emergência nas comunidades ${ }^{69}$ ou de curto prazo (Educação "Just in Time”), realizados durante ou após o evento, em poucos dias ou horas ${ }^{70}$.

Outro tema relacionado à educação foi o de conscientização e sensibilização pública. Nos períodos pré, durante e após enchentes a conscientização e a sensibilização publica são também indispensáveis na prevenção dos riscos e minimização dos danos, sendo destacados os sistemas de alerta e de comunicação contextualizados sobre os tipos de enchentes e considerando os grupos vulneráveis ${ }^{7,24,32}$. Os temas envolvidos nestes processos podem ser desde procedimentos para o cuidado com o consumo de água e alimentos e a higienização dos mes$\operatorname{mos}^{8,11,23,32,34,38,39,47,60}$, uso de inseticidas domésticos para o controle de vetores ${ }^{33}$ ou mesmo temas bastante específicos, como ações necessárias em casos de contaminação química (prevenção, descontaminação e tratamento $)^{39}$, utilizando desde de panfletos e alto-falantes, materiais informativos e didáticos, aos meios de comunicação de massa ${ }^{11,39,52,71}$. Podem envolver a preparação das comunidades, nas casas, escolas e locais de trabalho, para alerta (evacuações e rotas de fuga, zonas de enchentes, não dirigir carros e motos nem bicicletas em áreas inundadas, etc.) e respostas comunitárias ${ }^{24,67}$. Além disto, a população deve ser informada sobre os progressos nas ações de mitigação das consequências das enchentes, seja pelos órgãos governamentais ou por grupos de voluntários ${ }^{20,52,72}$.

A conscientização e a sensibilização pública não podem ser dissociadas de processos diretamente orientados para os profissionais do setor saúde. Informações e relatos de experiências de uma localidade podem servir para conscientizar, sensibilizar e treinar profissionais de outras que vivenciam problemas de saúde similares, como, por exemplo, as relacionadas a primeiros socorros e orientações sobre tratamento e prevenção de doenças relativas a enchentes como leptospirose e malária ${ }^{1732,52}$. Estes relatos e experiências, além de nortearem processos de conscientização, sensibilização e treinamento, também devem se converter em guias de respostas do setor saúde para situações de emergência, que podem envolver, inclusive, situações de maior complexidade, como a contaminação química que ocorre em eventos de enchentes ${ }^{39}$.

Este universo de ações e respostas do setor saúde exige o planejamento e a cooperação com outros setores, de modo conjunto e coordenado (centros de controle de operações) com outros setores governamentais (defesa civil, meio ambiente, forças armadas, agricultura, comunicação, transportes, polícia, etc.) e em diferentes níveis (municipais, estaduais e federais), além de empresas privadas (comunicação, transportes, etc.) e organizações não-governamentais, para prevenção e mitigação dos impactos das enchentes, bem como assistência e cuidado aos afetados, como, por exemplo, arrecadação de fundos e

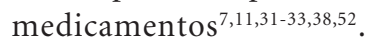

A cooperação com outros setores é fundamental, pois após algumas enchentes são neces- 
sárias medidas de reparo estrutural devido aos danos materiais, como providenciar reparos nas ruas, avenidas, pontes, e nos serviços médicos de emergência, restaurando e melhorando as condições originais locais ${ }^{20,33}$. É necessário ter equipamentos de emergência como geradores de energias e bombas de água, assim como outras estratégias que permitam tanto restaurar o funcionamento dos serviços (incluindo os de saúde), como minimizar os impactos dos desabrigados, permitindo o quanto antes a volta à sua rotina normal de atividades, diminuindo os impactos na saúde das vitimas ${ }^{24,33}$.

\section{Discussão}

Embora o presente universo de pesquisa tenha se restringindo a 70 artigos, considerou-se que o tipo de abordagem adotada para a análise dos mesmos propiciou um conjunto de resultados abrangentes sobre causas, consequências e respostas e ações não encontrado em nenhum dos artigos incluídos, no qual cerca de $3 / 4$ eram oriundos de instituições de países desenvolvidos, como América do Norte e Europa, Austrália e Japão.

Em relação às causas identificadas e sistematizadas no Quadro 1, é interessante observar que a grande maioria atribui às mudanças climáticas e ao aquecimento global os principais fatores na origem das enchentes. Se por um lado não se pode deixar de considerar a importância destes fatores no aumento da frequência e intensidade das chuvas, é necessário observar que ao tornar estes fenômenos como causas das enchentes, mesmo reconhecendo que tenham como principal força motriz atividades humanas (produção e consumo de energia, desmatamentos, queimadas, etc.), acabam por tornar distantes os processos de determinação social da exposição e as consequências destes eventos nos espaços geográficos em que vivem e trabalham as populações afetadas. Corresponde ao que Natenzon ${ }^{73}$ denomina de "naturalização" do processo social de causa das enchentes. Interessante observar se for tomado como exemplo o relatório do EIRD sobre redução do risco de desastres no mundo ${ }^{2}$, para as causas subjacentes das enchentes encontram-se as mudanças climáticas como apenas um fator entre tantos outros, como condições de vida, sistema de proteção social, planejamento urbano e degradação ambiental, para citar alguns exemplos.

No Quadro 2 estão sistematizadas as consequências ambientais em três grupos, sendo que dentre estes, a grande maioria destacou a conta- minação de duas fontes vitais para a saúde humana: águas e alimentos. A questão é que a contaminação destas duas fontes vitais, assim como de outras (solo, por exemplo), não se encontra dissociada do comprometimento e/ou ruptura de serviços fundamentais, como os de saneamento. Se for considerado que as enchentes apenas agravam condições de vida e saúde já existentes nos contextos em que ocorrem, se estas são vulneráveis, maiores serão as consequências que provocarão. Apesar do reconhecimento da vulnerabilidade como uma condição que tornam as consequências das enchentes sobre determinados grupos populacionais, comunidades ou mesmo países mais graves ${ }^{5,9,18,37}$, há ainda carência na literatura de análises que incorporem de modo sistemático conceitos e métodos que permitam melhor compreender e analisar os processos de determinação das vulnerabilidade social e ambiental às enchentes, como propõem autores argentinos ${ }^{73}$ e brasileiros ${ }^{74}$.

Se grande parte das consequências ambientais acaba por se concentrar nas que resultarão principalmente em doenças infecciosas e parasitárias, seja pela contaminação de águas e alimentos, seja pela alteração dos ciclos de hospedeiros e vetores, não se pode deixar de observar que as consequências sobre a saúde vão muito além destas, como se pode observar no Quadro 3. A demora nos processos de reabilitação e reconstrução para o restabelecimento da infraestrutura, serviços e economia local (Quadro 4) podem ampliar os espectro de doenças e agravos (transtornos mentais, lesões e causas externas, para citar alguns exemplos) ou agravar várias das já existentes (crônica e degenerativas), ampliando ainda mais os impactos das mesmas e tornando crônicas as consequências de um evento agudo. Este é tema que merece ampla investigação como o que relaciona a vulnerabilidade social aos desastres, envolvendo a precariedade nas ações e respostas institucionais para a redução dos ris$\cos ^{18,73}$, pois o que se tem não é somente o aumento da mortalidade nos meses subsequentes ao evento ${ }^{7,11,18}$, mas também a ampliação e o agravamento de doenças pela demora nos processos de reabilitação e reconstrução.

A questão das respostas e ações para prevenção e mitigação das consequências das enchentes, principalmente pelo setor saúde, surge como um tema fundamental. Mas para que sejam efetivas torna-se necessário programar e articular as ações capazes de promover avaliações rápidas, diagnosticando situações relativas a vigilância em saúde (diagnostico da área afetada, do 
risco sócio ambiental e da situação epidemiológica, vigilância da qualidade da água para consumo humano e alimentos), articuladas com ações e medidas de prevenção e controle de hospedeiros e vetores, vacinações, atenção e cuidados, com as outras ações voltadas para a prevenção e o alerta de desastres e sistematizadas no Quadro 5. Além disto, estas respostas e ações do setor saúde sistematizadas a partir desta revisão, e em grande parte também encontradas nos documentos nacionais que orientam as ações do Ministério da Saúde ${ }^{75}$, para que tenham maior alcance, necessitam estar estreitamente articuladas com as medidas de prevenção e mitigação dos desastres realizadas por outros setores, desde medidas de prevenção da exposição da população antes das enchentes e os sistemas de alerta nos períodos imediatamente anteriores às mesmas, até os processos de restabelecimento e recuperação da infraestrutura após o evento.

Por exemplo, a experiência sobre desastres recentes no Brasil, atingindo estados como Santa Catarina, (2008, 135 óbitos), Alagoas e Pernambuco (2010, 50 óbitos) e Região Serrana do Rio de Janeiro (2011, 918 óbitos) apontam que embora distintas, as ações de prevenção devem combinar medidas intersetoriais sobre uso e ocupação do solo. Isto envolve medidas sobre a ocupação de margens de rios preservando as áreas de proteção permanente e as unidades de conservação, as políticas de gestão da ocupação das áreas urbanas e das margens de rios combinadas com as de habitação evitando construções inadequadas próximas aos rios e áreas de encosta, drenagem e dragagens de rios, coleta e disposição adequada de resíduos e entulhos, reflorestamento e revitalização dos cursos d'água, entre outras ${ }^{7-78}$.

Outra questão essencial para a prevenção aos desastres é a capacidade de produzir e disseminar de modo articulado informações para o alerta antecipado que permitam atuar de modo integrado em um tipo de desastre que se caracteriza pela sazonalidade. Isto envolve tanto informações climáticas e meteorológicas, como sistemas de monitoramento e vigilância das enchentes, de modo a propiciar uma melhor preparação das ações e respostas da saúde pública.

\section{Considerações finais}

As enchentes encontram-se entre os desastres naturais que se caracterizam por alta frequência e baixa severidade em termos de óbitos, mas sendo responsáveis por grande proporção de danos à infraestrutura local, às habitações e às condições de vida das comunidades e das sociedades de baixa renda. Isto não exclui a possibilidade de que, principalmente por incapacidade de desenvolver estratégias de prevenção e mitigação, ocorram eventos com grande número de óbitos, como nos casos exemplificados de Santa Catarina e Rio de Janeiro.

As tendências atuais de crescimento e concentração da população em áreas urbanas, sem a adequada infraestrutura, e com degradação ambiental e desigualdades sociais já apontam para o crescimento das populações expostas e das perdas econômicas relacionadas às enchentes, isto mesmo sem considerar que a intensificação das mudanças climáticas representarão o aumento na frequência e na gravidade de eventos como esses ${ }^{79}$. Neste cenário, é previsto que as populações mais vulneráveis e menos preparadas sofram cada vez mais as consequências.

O país deve estar cada vez mais preparado para reduzir os riscos atuais e futuros. Assim, tomando como referência o documento do Secretariado das Nações Unidas para a Rio+20, sobre Redução de Riscos de Desastres e Construção da Resiliência ${ }^{80}$, considerou-se que a redução das causas e consequências das enchentes no âmbito do setor saúde deve envolver respostas integradas com amplas políticas para o desenvolvimento sustentável, reduzindo a vulnerabilidade de determinadas localidades e populações. Neste artigo, ao procurar sistematizar e sintetizar as causas, as consequências e as respostas obtidas com o objetivo de oferecer subsídios para uma melhor compreensão destes eventos, que embora nem sempre sejam catastróficos, respondem por impactos recorrentes sobre a saúde e a perda da qualidade de vida, exigindo abordagens integradas. 


\section{Colaboradores}

CM Freitas e EF Ximenes participaram igualmente de todas as etapas de elaboração do artigo.

\section{Referências}

1. United Nations Conference on Sustainable Development (UNCSD). Disaster-resilient Societies - Facts and figures. [documento da internet]. 2012 [acessado 2012 jan 12], [cerca de 2 p.]. Disponível em: http://www.un.org/en/sustainablefuture/disasters. shtml

2. International Strategy for Disaster Reduction (ISDR). Global Assessment Report on Disaster Risk Reduction - Risk and poverty in a changing climate Invest today for a safer tomorrow. Geneva: United Nations; 2009.

3. Blashki G, McMichael T, Karoly DJ. Climate change and primary health care. Aust Fam Physician 2007; 36(12):986-989.

4. Greenough G, McGeehin M, Bernard SM, Trtanj J, Riad J, Engelberg D. The potential impacts of climate variability and change on health impacts of extreme weather events in the United States. Environmental Health Perspectives 2001; 109(Supl. 2):191198.

5. Haines A, Kovats RS, Campbell-Lendrum D, Corvalan C. Climate change and human health: impacts, vulnerability and public health. Public Health 2006; 120(7):585-596.

6. Wang Y. Environmental degradation and environmental threats in China. Environmental Monitoring and Assessment 2004; 90(1-3):161-169.

7. Fundter DQ, Jonkman B, Beerman S, Goemans CL, Briggs R, Coumans F, Lahaye JW, Bierens J. Health impacts of large-scale floods: governmental decision-making and resilience of the citizens. Prehosp Disaster Med 2008; 23(4):s70-73.

8. Ebi KL, Paulson JA. Climate change and children. Pediatr Clin North Am 2007; 54(2):213-226.

9. Haines A, Kovats RS, Campbell-Lendrum D, Corvalan C. Climate change and human health: impacts, vulnerability, and mitigation. Lancet 2006; 367(9528):2101-2109. (Erratum in: Lancet 2006; 368 (9536):646).

10. Faergeman O. Climate change and preventive medicine. Eur J Cardiovasc Prev Rehabil 2007; 14(6): 726-729.

11. Kovats RS. Will climate change really affect our health? Results from a European Assessment. $\mathrm{J} \mathrm{Br}$ Menopause Soc 2004; 10(4):139-144.

12. Majra JP, Gur A. Climate change and health: Why should India be concerned? Indian J Occup Environ Med. 2009; 13(1):11-16.

13. Abaya SW, Mandere N, Ewald G. Floods and health in Gambella region, Ethiopia: a qualitative assessment of the strengths and weaknesses of coping mechanisms. Global Health Action 2009; 2. [acessado 2012 maio 2]. Disponível em: http://www.global health action.net/index.php/gha/article/view/2019/2484

14. Bich TH, Quang LN, Ha le TT, Hanh TT, GuhaSapir D. Impacts of flood on health: epidemiologic evidence from Hanoi, Vietnam. Glob Health Action. 2011; 4. [acessado 2012 maio 2]. Disponível em: http://www.globalhealthaction.net/index.php/gha/ article/view/6356 
15. Sayre L, Rhazi N, Carpenter H, Hughes NL. Climate change and human health: the role of nurses in confronting the issue. Nurs Adm Q 2010; 34(4):334-342.

16. George P. Health impacts of floods. Prehosp Disaster Med. 2011; 26(2):137. (Comment on Prehosp Disaster Med. 2010; 25(3):265-272).

17. Kshirsagar NA, Shinde RR, Mehta S. Floods in Mumbai: impact of public health service by hospital staff and medical students. J Postgrad Med 2006; 52(4):312-314.

18. Tapsell SM, Penning-Rowsell EC, Tunstall SM, Wilson TL. Vulnerability to flooding: health and social dimensions. Philos Transact A Math Phys Eng Sci 2002; 360(1796):1511-1525.

19. Booker SM. Evaluating Floyd's Effect on Health in Eastern North Carolina. Environ Health Perspect 2000; 108(2):A67.

20. Cervenka J. Health aspects of Danube river floods. Ann Soc Belg Med Trop 1976; 56(4-5):217-222.

21. Ivers LC, Ryan ET. Infectious diseases of severe weather-related and flood-related natural disasters. Current Opinion in Infectious Disease 2006; 19(5):40814.

22. Middleton J. Environmental health, climate chaos and resilience. Medicine, Conflict and Survival 2008; 24 (Supl. 1):S62-79.

23. Miettinen IT, Zacheus $\mathrm{O}$, von Bonsdorff $\mathrm{CH}$, Vartiainen T. Waterborne epidemics in Finland in 1998-1999. Water Sci Technol 2000; 43(12):67-71.

24. Keim M E. Building human resilience: the role of public health preparedness and response as an adaptation to climate change. Am J Prev Med 2008; 35(5):508-516.

25. Ebi KL, Mills DM, Smith JB, Grambsch A. Climate change and human health impacts in the United States: an update on the results of the U.S. national assessment. Environmental Health Perspectives 2006; 114(9):1318-1324.

26. Chan E, Griffiths S. The implication of water on public health: the case of China. Perspect Public Health 2010; 130(5):209-210.

27. Kovats RS, Bouma MJ, Hajat S, Worrall E, Haines A. El Niño and health. Lancet 2003; 362(9394):14811489.

28. Githeko AK, Lindsay SW, Confalonieri UE, Patz JA. Climate change and vector-borne diseases: a regional analysis. Bull World Health Organ 2000; 78(9): 1136-1147.

29. Morrow RC, Llewellyn DM. Tsunami overview. Military Medicine 2006; 171(10 Supl. 1):5-7.

30. Adel MM. Effect on water resources from upstream water diversion in the Ganges basin. J Environ Qual 2001; 30(2):356-368

31. Clinton JJ, Hagebak BR, Sirmons JG, Brennan JA. Lessons from the Georgia floods. Public Health Rep 1995; 110(6):684-688.

32. White K. Signs of a olive branch: confronting the environmental health consequences of the Midwestern floods. Environ Health Perspect 1993; 101(7):584588.

33. Von Zuben Junior FJ, Hayes Junior GR, Anderson EC. Public health disaster aid in Rio Grande flood of 1954. Public Health Rep. 1957; 72(11):1009-1017.

34. Hiscock IV. Flood disaster and Public health. Can J Public Health 1956; 47(10):420-426.
35. Noji EK. The Public Health Consequences of Disaster. Prehosp Disaster Med 2000; 15(4):147-157.

36. Kupek E, Faversani MCSS, Philippi JMS. The relationship between Rainfall and Human leptospirosis in Florianopolis, Brazil, 1991-1996. Braz J Infect Dis 2000; 4(3):131-134.

37. Kim ES, Choi HI. Assessment of vulnerability to extreme flash floods in design storms. Int J Environ Res Public Health 2011; 8(7):2907-2922.

38. Elliot GR, Bowering R, Frith M. Public health Services in British Colombia during the Floods of 1948. Can J Public Health 1949; 40(9):371-381.

39. Euripidou E, Murray V. Public health impacts of floods and chemical contamination. J Public Health (Oxf) 2004; 26(4):376-383.

40. Wolfe AH, Patz JA. Reactive Nitrogen and Human Health: Acute and Long-term Implications Ambio 2002; 31(2):120-125.

41. Taylor J, Lai KM, Davies M, Clifton D, Ridley I, Biddulph P. Flood management: prediction of microbial contamination in large-scale floods in urban environments. Environ Int 2011; 37(5):1019-29.

42. Satterthwaite D. The Impact on health of urban environment. Environ Urban 1993; 5(2):87-111.

43. Brandt M, Brown C, Burkhart J, Burton N, CoxGanser J, Damon S, Falk H, Fridkin S, Garbe P, McGeehin M, Morgan J, Page E, Rao C, Redd S, Sinks T, Trout D, Wallingford K, Warnock D, Weissman D. Mold prevention strategies and possible health effects in the aftermath of hurricanes and major floods. MMWR Recommendations and Reports 2006; 55(RR-8):1-27.

44. Seto EY, Wu W, Liu HY, Chen HG, Hubbard A, Holt A, Davis GM. Impact of changing water levels and weather on Oncomelania hupensis hupensis populations, the snail host of Schistosoma japonicum, downstream of the Three Gorges Dam. Ecohealth 2008; 5(2):149-158.

45. Feng S, Tan H, Benjamin A, Wen S, Liu A, Zhou J, Li S, Yang T, Zhang Y, Li X, Li G. Social support and posttraumatic stress disorder among flood victims in Hunan, China. Ann Epidemiol 2007; 17(10): 827-833.

46. Kunii O, Nakamura S, Abdur R, Wakai S. The impact on health and risk factors of the diarrhoea epidemics in the 1998 Bangladesh floods. Public Health 2002; 116(2):68-74.

47. Srinivasan S. After the floods: health services' responsibilities in a crisis. Indian J Med Ethics 2005; 2(4):108-109.

48. Guha-Sapir D. Rapid assessment of health needs in mass emergencies: review of current concepts and methods. World Health Stat Q 1991; 44(3):171-181.

49. Quinlisk P, Jones MJ, Bostick NA, Walsh LE, Curtiss R, Walker R, Mercer S, Subbarao I. Results of Rapid Needs Assessments in Rural and Urban Iowa Following Large-scale Flooding Events in 2008. Disaster Med Public Health Prep 2011; 5(4):287-292.

50. Warraich H, Zaidi AK, Patel K. Floods in Pakistan: a public health crisis. Bull World Health Organ 2011; 89:236-237.

51. Du W, FitzGerald GJ, Clark M, Hou XY. Health impacts of floods. Prehosp Disaster Med. 2010; 25(3):265-272. 
52. Dai ZC. No epidemics despite devastating floods. Chin Med J (Engl) 1992; 105(7):531-534.

53. Zhou J, Huang X, He H, Zhang X, Liu A, Yang T, Li S, Tang X, Tan H. Epidemiological Study on leptospirosa infection of host animals and health population in flood areas. Zhong Nan Da Xue Xue Bao Yi Xue Ban. 2009; 34(2):99-103.

54. Hsu NY, Chen PY, Chang HW, Su HJ. Changes in profiles of airborne fungi in flooded homes in southern Taiwan after Typhoon Morakot. Sci Total Environ 2011; 409(9):1677-1682.

55. Barbeau DN, Grimsley LF, White LE, El-Dahr JM, Lichtveld M. Mold exposure and health effects following hurricanes Katrina and Rita. Annu Rev Public Health 2010; 31:165-178.

56. Curtis L, Ross M, Scheff P, Persky V, Wadden R, Ramakrishnan V, Hryhorczuk D. Dust-mite-allergen concentrations in asthmatics' bedrooms in the Quad Cities (Illinois, USA) after the Mississippi River floods of 1993. Allergy 1997; 52(6):642-649.

57. Huang P, Tan H, Liu A, Feng S, Chen M. Prediction of posttraumatic stress disorder among adults in flood district. BMC Public Health 2010; 10: 207.

58. Paranjothy S, Gallacher J, Amlôt R, Rubin GJ, Page L, Baxter T, Wight J, Kirrage D, McNaught R, Palmer SR. Psychosocial impact of the summer 2007 floods in England. BMC Public Health 2011; 11:145.

59. Vineis P. Climate change and the diversity of its health effects. Int J Public Health 2010; 55(2):81-82.

60. Gautman K. Organizational problems Faced by the Missouri DOH in providing Disaster Relief during the 1993 Floods. J Public Health Manag Pract 1998; 4(4):79-86.

61. Diaz JH. Global climate changes, natural disasters, and travel health risks. J Travel Med 2006; 13(6):361372 .

62. Woods CE, Goodman D, Mills J, Usher K, McBride WJ. Weather to evacuate? Med J Aust 2011; 195(1112):712-713.

63. Bich T, Quang L, Thanh HA L, Duc T, Guha-Sapir D. Impacts of flood on health: epidemiologic evidence from Hanoi, Vietnam. Global Health Action 2011. [acessado 2012 maio 2]. Disponível em: http:/ /www.globalhealthaction.net/index.php/gha/article/view/6356

64. Tomio J, Sato H, Mizumura H. Interruption of medication among outpatients with chronic conditions after a flood. Prehosp Disaster Med. 2010; 25(1):42-50.

65. Thomson MC, Connor SJ, Zebiak SE, Jancloes M, Mihretie A. Africa needs climate data to fight disease. Nature 2011; 471(7339):440-442.

66. Fleming RM. When floods came. Public Health Nurs 1952; 44(11):636-637.

67. Centers for Disease Control and Prevention (CDC). Flood-Related Mortality - Missouri, 1993. MMWR Morb Mortal Wkly Rep 1993; 42(48): 941-943.

68. Rowland MG. The Gambia and Bangladesh, the seasons and diarrhea. Dialogue Diarrhoea 1986; (26):3.

69. Pfeiffer C, Glaser S, Vencatesan J, SchliermannKraus E, Drescher A, Glaser R. Facilitating participatory multilevel decision-making by using interactive mental maps. Geospatial Health 2008; 3(1): 103-112.
70. Ardalan A, Linkov F, Shubnikov E, LaPorte RE. Public awareness and disaster risk reduction: justin-time networks and learning. Prehospital and $\mathrm{Di}$ saster Medicine 2008; 23(3):286-288.

71. Murphy MW, Iqbal S, Sanchez CA, Quinlisk MP. Postdisaster health communication and information sources: the Iowa flood scenario. Disaster Med Public Health Prep. 2010; 4(2):129-134.

72. Gaur SD, Marwah SM. Public health aspects of floods with illustrations from 1967 Varanasi floods. Indian J Public Health 1968; 12(2):93-94.

73. Natenzom C. Vulnerabilidad, incertitumbre y planificación participativa de desastres: el caso de las inundaciones catastróficas en Argentina. In: Climate change impacts and integrated assessment EMF Workshop IX; 2003 Jul 28-Aug 7; Snowmass, Colorado, EUA.

74. Alves HPF. Vulnerabilidade socioambiental na metrópole paulistana: uma análise sociodemográfica das situações de sobreposição espacial de problemas e riscos sociais e ambientais. Rev Brasileira de Estudos Populacionais 2006; 23(1):43-59.

75. Brasil. Ministério da Saúde (MS). Secretaria de Vigilância em Saúde (SVS). Plano de contingência de vigilância em saúde frente a inundações. Brasília: MS, SVS; 2005.

76. Brasil. Ministério do Meio Ambiente (MMA). Secretaria de Biodiversidade e Florestas (SBF). Relatório de Inspeção Área atingida pela tragédia das chuvas na Região Serrana do Rio de Janeiro - Áreas de Preservação Permanente, Unidades de Conservação \& Áreas de Risco - O que uma coisa tem a ver com a outra? Brasília: MMA, SBF; 2011.

77. Universidade Federal de Santa Catarina. Centro Universitário de Estudos e Pesquisas sobre Desastres (UFSC/CEPED). Diretrizes em redução de riscos de desastres: Região Serrana do Rio de Janeiro. Florianópolis: CEPED, UFSC; 2011.

78. Santa Catarina. Secretaria de Estado do Desenvolvimento Econômico Sustentável de Santa Catarina, Fundação de Apoio à Pesquisa Científica e Tecnológica do Estado de Santa Catarina. Plano Integrado de Prevenção e Mitigação de Riscos de Desastres Naturais na Bacia Hidrográfica do Rio Itajaí. Santa Catarina. SEDES-SC, FAPESC; 2009.

79. World Bank (WB), United Nations (UN). Natural hazards, unnatural disasters: the economics of effective prevention. Washington DC: WB, UN; 2010.

80. United Nations Conference on Sustainable Development (UNCSD). Disaster Risk Reduction and Resilience Building. [documento da internet]. 2012 [acessado 2012 jan 12], [4 p.]. Disponível em: http:/ /www.uncsd2012.org/rio20/index.php?page $=$ view \& $\mathrm{nr}=225 \&$ type $=400 \&$ menu $=45$

Artigo apresentado em 05/04/2012

Aprovado em 10/04/2012

Versão final apresentada em 15/04/2012 